\title{
AN 8 GEV H- MULTI-TURN INJECTION SYSTEM FOR THE FERMILAB MAIN INJECTOR*
}

\author{
D.E. Johnson", P. Yoon, FNAL, Batavia, IL, USA \\ C-J. Liaw, D. Raparia, J. Bebee-Wang, BNL, Upton, NY , U.S.A.
}

\begin{abstract}
An $8 \mathrm{GeV}$ superconducting linear accelerator (SCL) has been proposed [1] as a single stage $\mathrm{H}^{-}$injector into the Main Injector (MI) synchrotron . This would be the highest energy $\mathrm{H}$ - multi-turn injection system in the world. The conceptual design of an injection system has been further refined by addressing transverse phase space painting issues, chicane dipole fields and foil location, foil temperature issues, and initial longitudinal phase space painting simulations. We present the current state of design.
\end{abstract}

\section{ACCEPTANCE AND BEAM LOSS}

During the design of the MI, the dynamic aperture was estimated to be $127 \pi$-mm-mr [2] based upon tracking and defined as the smallest amplitude particle at a beta of $70 \mathrm{~m}$ that did not survive 35000 turns at $8.9 \mathrm{GeV} / \mathrm{c}$, the expected injection dewll time. For $\mathrm{H}$ - painting, the expected dwell time is a factor of 10 to 100 smaller with the actual injection being no longer than $3 \mathrm{~ms}$ (270 turns), thus reducing sensitivities to magnet nonlinearities.

The MI utilizes an elliptical beam pipe $(61 \mathrm{~mm} \mathrm{x}$ $26.5 \mathrm{~mm}$ ID) throughout the $3.3 \mathrm{~km}$ with the exception of the RF and extraction regions. The limiting aperture is at the extraction Lambertsons where an acceptance of $80 \pi$ mm-mr will be assumed. [3]

The current operational criteria for losses in the MI during NuMI operations is 1 to 1.5 kJoule beam energy/cycle. Most loss is at injection energy and during the parabola. For an expected intensity of $1 \mathrm{E} 14 / \mathrm{sec}$ and ssuming no loss after $10 \mathrm{GeV} / \mathrm{c}$, then $1.5 \mathrm{~kJ}$ at a $1.5 \mathrm{sec}$ cycle time corresponds to 7.7E11 lost protons/sec or about 985 watts. A collimation system is being installed in the MI with a calculated efficiency of $99.8 \%$ capable of handling $1.6 \mathrm{~kW}$. [4]

\section{INJECTION OPTICS AND LAYOUT}

The MI-10 straight section design has been modified to a symmetric straight section with 38 meters of free space between the quad doublets [5]. The entire injection system (injection chicane, injection foil, and horizontal painting magnets) is contained within the straight. The injection chicane consists of four DC magnets which create an orbit offset from the straight section centerline. The magnitude of this orbit offset is constrained by the peak field in the second and third chicane magnets and physical constraints on the transport line and injection absorber.[6]

*Operated by Fermi Research Alliance, LLC under contract No. DEAC02 -7CH11359 with the United States Department of Energy \#dej@fnal.gov
The injected $\mathrm{H}$ - first sees the dipole field of the second chicane magnet, the final bend for the $\mathrm{H}$ - prior to the stripping foil. It's field is $\sim 500 \mathrm{G}$, with a stripping rate of $2.2 \mathrm{E}-10 / \mathrm{m}$, to prohibit any magnetic stripping.

The optimization of the field at the foil location, the peak dipole field and end field of the third chicane is in progress and has not been completed.

Location of the foil in the end field of the third chicane magnet at a magnitude of $\sim 600 \mathrm{G}$ will minimize any Lorentz stripping of the $\mathrm{H}$ - prior to the foil. The rising end field shape, $\mathrm{dBy} / \mathrm{dz}$, will determine the differential integrated field the excited states experience prior to decay compared to the integrated field the protons experience after the stripping foil. A flux catcher/ end field clamp is planned to rapidly terminate the field to reduce the end field.

The dipole field of the third chicane magnet should be either between the $n=4$ and $n=3$ states or between the $n=3$ and $\mathrm{n}=2$ states to inhibit stripping of the lower excited state. The current choice places the dipole field at about $5.5 \mathrm{kG}$ which is between the $\mathrm{n}=2$ and $\mathrm{n}=3$ Stark states such that the $n=1$ and $n=2$ stated are transported to the secondary foil for stripping and the resultant protons are transported to the injection absorber.

\section{Foil Stripping}

The current design utilizes a $425 \mu \mathrm{g} / \mathrm{cm}^{2}$ carbon foil which will produce the charge states shown in Table 1. The capture efficiency for $\mathrm{H}+$ is $97.76 \%$ with $2.24 \%$ in the form of $\mathrm{H} 0(n=1,2)$ being transported to the injection absorber. Increasing the foil thickness to $500 \mu \mathrm{g} / \mathrm{cm}^{2}$ increases capture efficiency and reduces the fraction of $\mathrm{n}=3$ and higher states and potential losses.

\section{Table 1}

\begin{tabular}{|l|l|l|}
\hline Charge state & $425 \mathrm{ug} / \mathrm{cm}^{2}$ & $500 \mathrm{ug} / \mathrm{cm}^{2}$ \\
\hline $\mathrm{H}^{+}$ & 97.6 & 99.0 \\
\hline $\mathrm{n}=1$ & 1.8 & 0.735 \\
\hline $\mathrm{n}=2$ & .45 & 0.245 \\
\hline $\mathrm{n}=3$ & .15 & .0068 \\
\hline $\mathrm{H}^{-}$ & .0022 & .0 .00028 \\
\hline Total $\mathrm{H}+$ captured & 97.76 & 99.02 \\
\hline
\end{tabular}

\section{Stripped electrons}

Stripped electrons will have the same velocity as $\mathrm{H}$ and as they travel toward the chicane magnet \#3 they will bend opposite direction of the protons. For chicane of $0.55 \mathrm{~T}$ and foils location of $0.06 \mathrm{~T}$, these electrons will have bending radius of about $5.5 \mathrm{~cm}$ and return back 
about $11 \mathrm{~cm}$ from the foil location in the horizontal plane where they can be collected by an electron catcher.

\section{TRANSVERSE PHASE SPACE PAINTING}

Transverse phase space painting will utilize a horizontal painting and vertical injection angle to produce a uniform phase space distribution [7]. A transverse (and longitudinal) phase space distribution at the foil has been produced from TRACK [8] by accelerating $45 \mathrm{~mA}$ in the linac, transporting through the beam transport line (including a debuncher) to the stripping foil. The final optics of the transport line was tuned to produce a spot size on the target of approximately $4 \mathrm{~mm}$ ( $3 \sigma$ ) in each dimension[9]. The program STRUCT [10] used this phase space an input to simulate the painting process in the absence of any space charge using the following horizontal and vertical waveforms.

$$
\begin{gathered}
B=B_{0}\left[\frac{R}{T}-\frac{P}{T}\left(1-\sqrt{\frac{2 n}{N}-\left(\frac{n}{N}\right)^{2}}\right)\right] \text { For } \mathrm{n}<\mathrm{N} \\
y^{\prime}=\left(\sigma_{44}^{c i r}-\sigma_{44}^{i n j}\right) \sqrt{\frac{2 N-n}{N}-\left(\frac{N-n}{N}\right)^{2}}
\end{gathered}
$$

where $\mathrm{B} 0$ is maximum kicker field, $\mathrm{P}$ is the painting displacement, $\mathrm{R}$ is the removal displacement, $\mathrm{T}=\mathrm{R}+\mathrm{P}, \mathrm{N}$ is the total number of painting turns, and $n$ is the injection turn number. In addition magnet nonlinearities have yet to be included in the simulation. Both of these effects will tend to increase the final painted emittance.

Based upon the current NuMI upgrade path emittance assumptions the simulation paints to $25 \pi$ with the potential of painting to larger emittancestaying within the machine acceptance and reducing the number of hits on the foil by circulating beam.

The final distribution after 270 turn 3 ms. injection is shown in Figure 1. This distribution corresponds to $+/-3 \sigma$ of a $25 \pi$-mm-mr normalized emittance at a horizontal $\beta$ of $70 \mathrm{~m}$ and vertical $\beta$ of $30 \mathrm{~m}$.
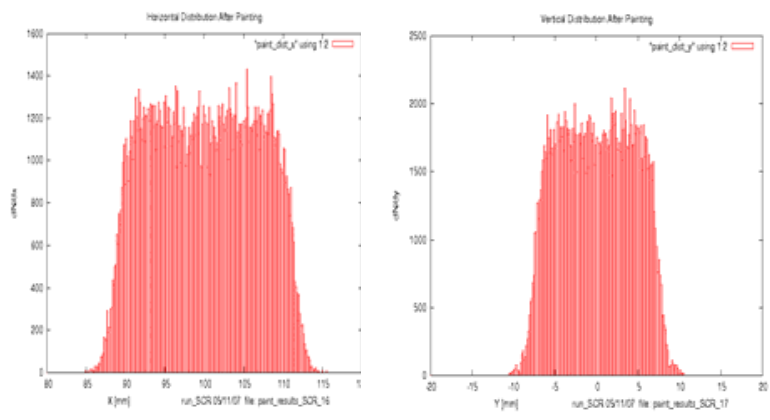

Figure 1. Resultant distribution

\section{INJECTION FOIL ISSUES}

\section{Foil Heating}

A simplified model [11] was used to determine the maximum foil temperature for various injection times and input beam sigma's on the foil. A total of 1.54E15 Hparticles were assumed each injection. Two injection periods were addressed, $1.5 \mathrm{sec}$ and $10 \mathrm{~Hz}$. Figure 2 shows seven injection pulses for the case of $3 \mathrm{~ms}$ injection (270 turns) at a rep rate of $1.5 \mathrm{sec}$ and 9 secondary hits/particle. The three curves represent three different incoming beam sigma's, $1 \mathrm{~mm}$ (black), $1.5 \mathrm{~mm}$ (red), and $2 \mathrm{~mm}$ (blue). Here, the rep rate is slow enough that the peak temperature decays back to ambient between pulses.

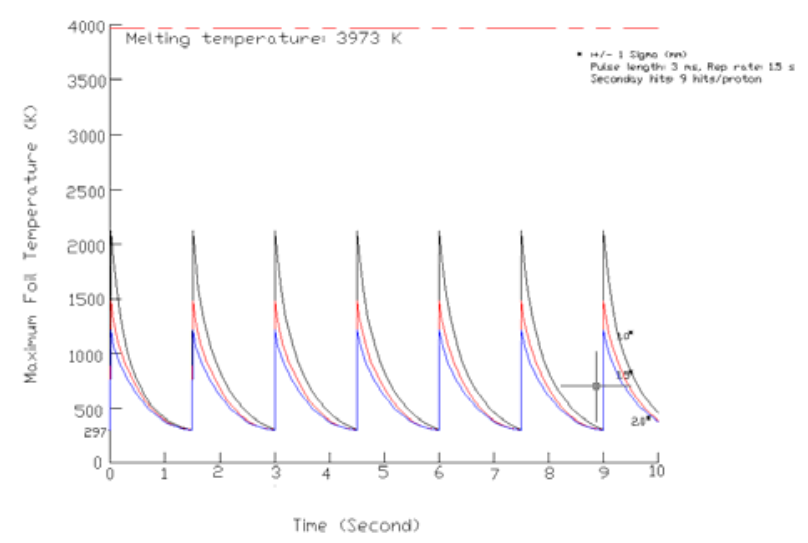

Figure 2. Peak foil temperature.

Figure 3 shows two temperature distributions for the case of a $1 \mathrm{~mm}$ sigma for the incoming $\mathrm{H}-$. The plot on the left only includes the contribution from the first turn $\mathrm{H}$ - (proton+two electrons) with a peak of $1881{ }^{\circ} \mathrm{K}$, while the picture on the right shows the contribution of the circulating beam with 9 hits/proton with a peak of 2115 ${ }^{\circ} \mathrm{K}$. The foil support is not shown and to the right $(+\mathrm{x})$.
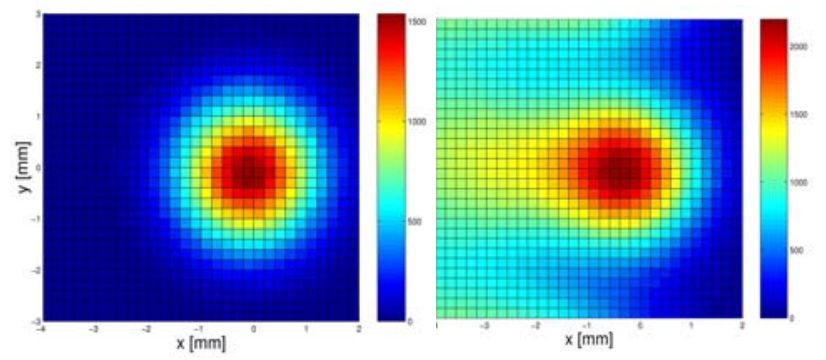

Figure 3. Foil temperature distribution for

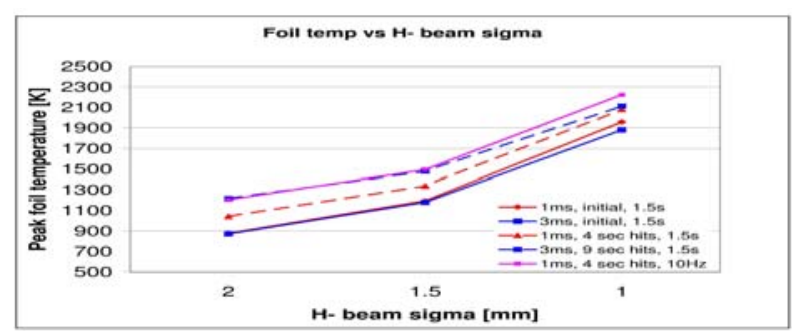

Figure 4. Summary of peak foil temperature 
Figure 4 shows a summary of the peak temperatures for the five different cases as a function of input beam sigma. The impact of input beam size and secondary hits can clearly be seen.Based upon these calculations an input $\mathrm{H}$ sigma in the range of 1.2 to $1.5 \mathrm{~mm}$ has been selected to keep a working temperature in the 1700 to $1800{ }^{\circ} \mathrm{K}$ range.

\section{LONGITUDINAL PHASE SPACE PAINTING}

The MI Rf frequency at injection is $52,811,400 \mathrm{~Hz}$ and has a harmonic number of 588. A beam gap of roughly 42 buckets (800 ns) is required for the abort kicker rise time implies a MI bunch intensity of 2.82E11 in 546 bunches. The linac bunching frequency is $325 \mathrm{Mhz}$. A $325 \mathrm{Mhz}$ fast chopper [Ref] after the $2.5 \mathrm{MeV}$ RFQ will generate the required bunch structure for MI injection and abort gap preservation. The ratio of the Linac and MI RF is $\sim 6.154$. Two of the six bunches are chopped In order to place the linac microbunches within $+/-6$ ns of the center of the MI bucket. The non-integer ratio also has the added advantage of parasitic longitudinal painting .

Initial ESME [12] simulations have investigated the utilization of a single and dual harmonic MI RF system. Simulations[13] using the single harmonic showed a peaked distribution. The second harmonic content of the dual harmonic system (400 kV on $53 \mathrm{Mhz}$ and $200 \mathrm{kV}$ on 106 Mhz system) was tuned to produce a more uniform

Her

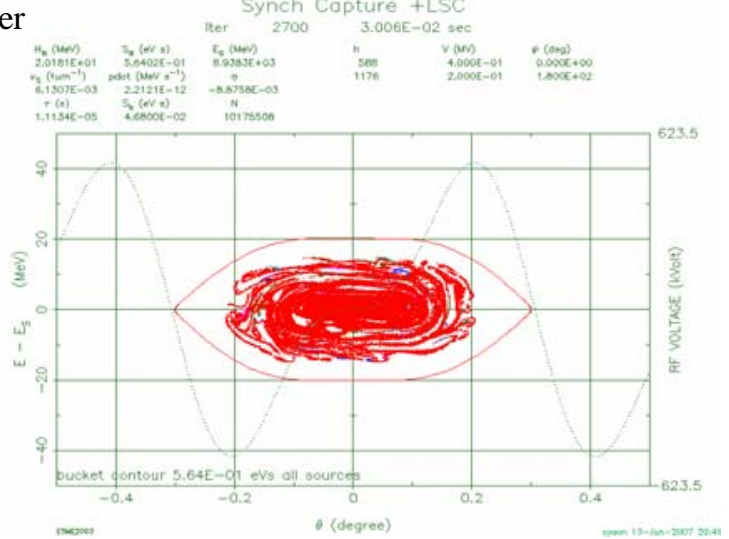

Figure 5: Longitudinal phase space after 270 turn microbunch injection and

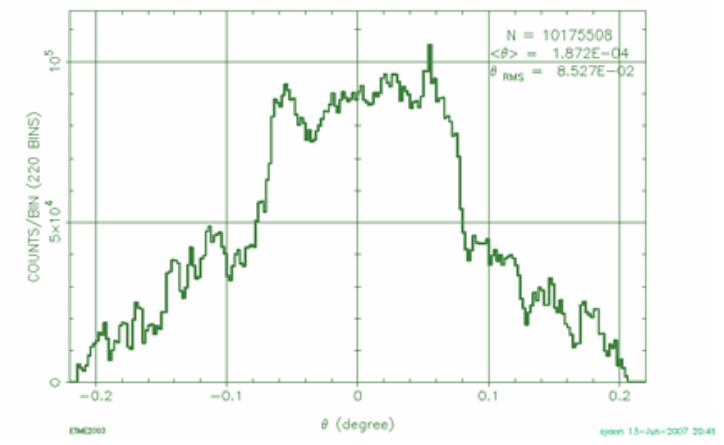

Figure 6: Longitudinal bunch density with dual harmonic RF system distribution after injection as shown in Figures 5 and 6. The final bunch length is +/- 0.2 degrees (12 ns) with an energy spread of $+/-10 \mathrm{MeV}$.

The current simulation includes longitudinal space chare and longitudinal impedance. Future simulations will include broadband impedance of the MI.

\section{SUMMARY}

We present the current state of the injection design for 8 $\mathrm{GeV} \mathrm{H}$ - into the Main Injector. Further optimizations will include chicane magnet design, estimation of activation in the injection region, and simulations that will include space charge and magnetic harmonics of the dipoles and quads

\section{REFERENCES}

[1] G. W. Foster, J. A. MacLachlan,”A Multi-mission 8 $\mathrm{GeV}$ Injector Linac as a Fermilab Booster Replacement”, Proceedings of LINAC2002, Gyeongju, Korea, p.826.

[2] Main Injector Technical Design Handbook, Chapter 2 page 10, August 1994.

[3] WeirenChou, et. al. "Operational Aspects of the Main Injector Large Aperture Quadrupole”, TUPAS014 this conference.

[4] A.I.Drozhdin, et.al., "Collimation System Design For Beam Loss Localization With Slip-stacking Injection in the Fermilab Main Injector”, TUPAS016 this conference.

[5] D. E. Johnson, "Design of an $8 \mathrm{GeV} \mathrm{H}$ - transport and Multi-Turn Injection System”, Proceedings of LINAC06, Nashville, Tenn., p. 779.

[6] D. E. Johnson, et.al., “A Conceptual Design of an Internal Injection Absorber for $8 \mathrm{GeV} \mathrm{H}$ - Injection into the Fermilab Main Injector”, TUPAS018 this conference.

[7] I.Sakai, et. al., "Phase-Space Painting for Charge Exchange Injection in the KEK Booster”, EPAC98, p.2137.

[8] P. Ostromov, et. "TRACK....

[9] Jean-Paul Carneiro, D. E. Jonson, "Start-to-End Simulations For the proponed Fermilab High Intensity Proton Source”, TUPAS012 this conference.

[10] A.I. Drozdhin, et. al. "STRUCT Progrsm User's Reference Manual”, $\quad$ http://wwwbd.fnal.gov/users/drozhdin/STRUCT

[11] C.J.Liaw, et. al., "Calculation of the Maximum Temperature on the Stripping Foil of the Spallation Neutron Source”, Proceedings of the 1999 Particle Accelerator Conference, NY. , page 3300.

[12] J. A. MacLachlan, "ESME Program Manual", http://www-ap.fnal.gov/ESME.

[13] P. S. Yoon, "8-GeV SCRF Linac andFMI Modeling”, http://wwwap.fnal.gov/ syoon/SC/FMI.html 
